\title{
Cast Shadow Detection for Surveillance System Based on Tricolor Attenuation Model
}

\author{
Linlin Zhu ${ }^{1, \text { a }}$, Jiandong Tian ${ }^{1, b}$, Yandong Tang ${ }^{1, \mathrm{c}}$, Jianda Han ${ }^{1, \mathrm{~d}}$ \\ State Key Laboratory of Robotics, Shenyang Institute of Automation, CAS \\ , Shenyang, 110016, PR China \\ zhulinlin@sia.cn, tianjd@sia.cn, ytang@sia.cn, jdhan@sia.cn
}

Keywords: Cast shadow, Target detection, Video surveillance, Tricolor attenuation model

\begin{abstract}
Shadows bring some undesirable problems in computer vision, such as object detecting in outdoor scenes. In this paper, we propose a novel method for cast shadow detecting for moving target in surveillance system. This measure is based on tricolor attenuation model, which describes the relationship of three color channel's attenuation in image when shadow happens. According to this relationship, the cast shadow is removed from the detected moving area, only the target area is left. Some experiments were done, and their results validate the performance of our method.
\end{abstract}

\section{Introduction}

Shadows, the common phenomena in most outdoor scenes, bring many problems. Cast shadow with moving target is one of these problems. Cast shadow detection is critical for accurate object detection in video streams since shadow points are often misclassified as object points. Shadows can cause object merging, object shape distortion, and even object losses, causing errors in segmentation and tracking. Figure 1 shows a target with cast shadow. Many algorithms have been proposed in the literatures that deal with shadow.

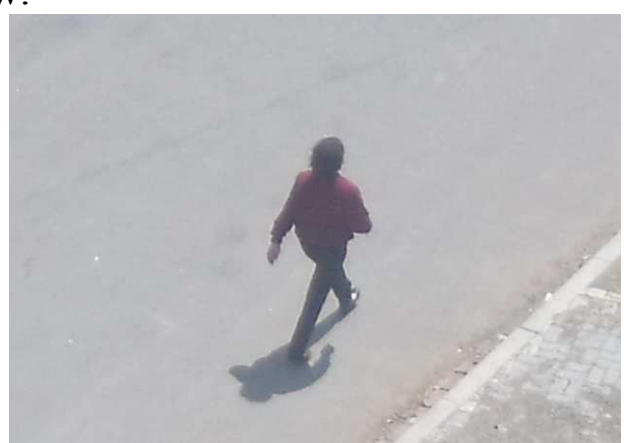

Fig. 1. A moving object with cast shadow

Technically, shadow detection methods can be classified into model-based and property-based. Model-based techniques need some prior knowledge, such as light and geometry [1], camera calibration [2], or indoor scenes [3]; model-based methods exploit some knowledge of the scene, which result in these methods being only used in the specific applications they are designed for. Property-based techniques indentify shadows by using shadow features. The most straightforward feature of a shadow is that it darkens the surface it cast on, and this feature is used by almost all methods. Other features like edge [4,5], histograms[6], texture[7], and color ratios[8] are also widely used. Sometimes, only one feature is not enough, most methods need to integrate more than one feature. Generally, property-based approaches are more flexible. They can be applied to a wider class of scenes and can be expanded to new applications.

Shadow is a half transparent area. Usually, the hue (color) of the surface which shadow cast on is considered doesn't change, which means that in RGB color space the attenuation of each channel is proportional. In fact, the attenuations in these three channels are not proportional strictly. In reference [9], Tian deduces a tricolor attenuate model (TAM) which describes the relationship of three color channel's attenuation in image when shadow happens. The main contribution of this paper is proposed a sample and effective method to remove cast shadow for surveillance. 
The rest of the paper is organized as follows. In section 2, we give a brief introduce and analysis of tricolor attenuation model. Based on this model, a cast shadow detect method is presented in section 3. In section 4, we give experimental results, followed by the conclusion in section 5 .

\section{Tricolor attenuation model}

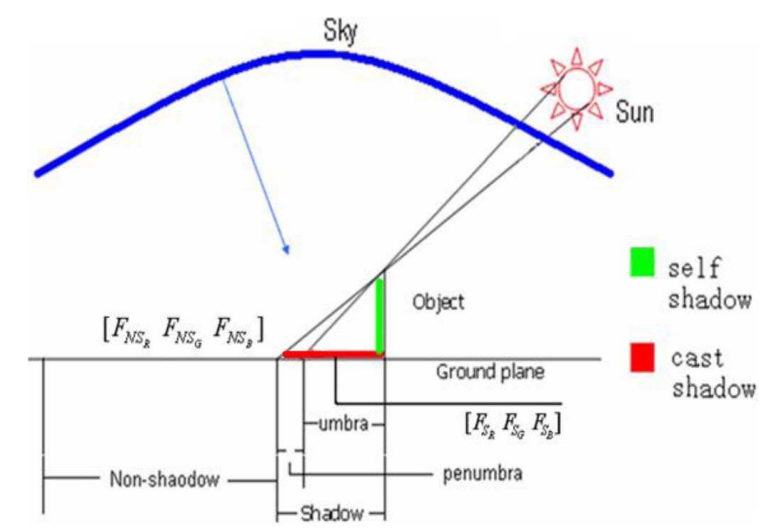

Fig. 2. Sketch map of shadow happens

The shadow will occur when direct light is occluded, and the illumination spectral power on object will attenuate. For a RGB tricolor image, it means that the pixel intensity values on shadow region will attenuate compared with its original intensity values without shadow. In reference [9], Tian proposed a shadow tricolor attenuation model for shadow detection in an image. Denoting $\left(F_{N S_{R}}, F_{N S_{G}}, F_{N S_{B}}\right)$ as the non-shadow pixel value vector of a RGB image $F, E_{d a y}(\lambda)$ and $E_{s k y}(\lambda)$ as the illumination spectral power distribution (SPD) of daylight and skylight respectively, where $E_{d a y}>E_{s k y}, \lambda$ is the wavelength. According to the tricolor attenuation model, the attenuation in each color channel satisfies

$$
\left[\begin{array}{c}
\Delta R \\
\Delta G \\
\Delta B
\end{array}\right]=\left[\begin{array}{c}
(\Delta R / \Delta B) \times \Delta B \\
(\Delta G / \Delta B) \times \Delta B \\
1 \times \Delta B
\end{array}\right]=\left[\begin{array}{c}
m \times\left(F_{N S_{R}} / F_{N S_{B}}\right) \\
n \times\left(F_{N S_{G}} / F_{N S_{B}}\right) \\
1
\end{array}\right] \cdot \Delta B
$$

where

$$
\left\{\begin{array}{l}
m=\frac{E_{d a y}\left(\lambda_{B}\right)}{E_{d a y}\left(\lambda_{R}\right)} \times \frac{E_{d a y}\left(\lambda_{R}\right)-E_{s k y}\left(\lambda_{R}\right)}{E_{d a y}\left(\lambda_{B}\right)-E_{s k y}\left(\lambda_{B}\right)} \\
n=\frac{E_{d a y}\left(\lambda_{B}\right)}{E_{d a y}\left(\lambda_{G}\right)} \times \frac{E_{d a y}\left(\lambda_{G}\right)-E_{s k y}\left(\lambda_{G}\right)}{E_{d a y}\left(\lambda_{B}\right)-E_{s k y}\left(\lambda_{B}\right)}
\end{array}\right.
$$

$m$ and $n$ are different with different sun angle, but when sun angle is less than 60 degree(most days of a year, from 9:00 am to 4:00 pm), $m$ and $n$ are quite stable: $\mathrm{m} \approx 1.31$ and $\mathrm{n} \approx 1.19$. In this paper we set $\mathrm{m}=1.31$ and $\mathrm{n}=1.19$.

The formula (1) implies the relationship of the attenuations in each color channel when shadow happens; we call this tricolor attenuation model. The disparities of channels of a shadow region are expected to be different from those of the corresponding non shadow background region. 


\section{Target detection based on TAM}

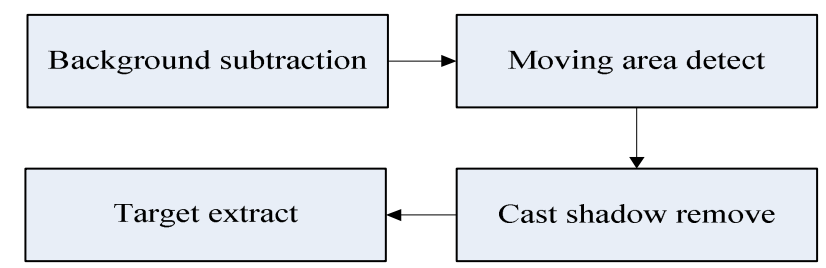

Fig. 3. Flow of moving target detect

Moving target detect method overview. Modeling and then finding deviations from the model for each incoming frame. Any significant change in an image region from the background model contains moving object. The pixels constituting the regions undergoing change are marked as foreground for further processing. Usually, these pixels whose change cause by cast shadow are also considered as foreground, and those pixels should be removed in object recognition and tracking. Therefore, as shown in Fig. 3, the moving target detect method in surveillance system can be divided into background subtraction, moving area detect, cast shadow remove and target extract. There are plenty literatures about background subtraction, we use the method which propose in reference [10] to model the background scene. And moving area can be found from the difference between current frame and the background model. This paper will focus on how to remove cast shadow from foreground which is described in next part.

Cast shadow detection based on TAM. According to the description in section 2, the pixels in cast shadow area have lower intensity value than background model; and the attenuation value in each channel in RGB space satisfied the relationship of equation(1). Based on these facts, we extract the target area without cast shadow in current frame as follow steps:

1) Given the background model I_background and current frame $I_{-}$current, calculate the difference by $I_{-}$diff $=I_{-}$current $-I_{-}$background;

2) Find the pixels whose difference exceed the set threshold as the moving area and the target candidate area $t_{-}$candidate;

3 ) Calculate the following variables for every pixel in $t_{-}$candidate

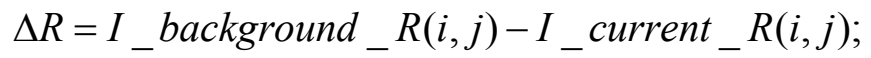

$\Delta G=I$ _background_G(i,j)-I_current_G(i,j);

$\Delta B=I$ _background_B(i,j)-I_current_B(i,j);

where $I_{\text {_background }} R$ means the intensity value of red channel in background model with biggest weight, similarity with others.

4) Based on TAM, if a pixel in cast shadow, the attenuation value in each color channel has certain rules. In our method, the pixels in $t_{-}$candidate and satisfied the eq (3) are consider as cast shadow. Then remove those pixels from foreground.

$$
\left\{\begin{array}{c}
\Delta R>0, \Delta G>0, \Delta B>0 \\
m * 0.8<\frac{I \_ \text {background_B }(i, j)}{I_{\text {_background_R }} R(i, j)} * \frac{\Delta R}{\Delta B}<m * 1.2 \\
n * 0.8<\frac{I_{-} \text {background_B(i,j) }}{I_{\text {_background_G }} G(i, j)} * \frac{\Delta G}{\Delta B}<n * 1.2
\end{array}\right.
$$

5 ) Extract the target from the left foreground by C-mean cluster method[11]. 


\section{Experiment Results}

We apply our method to remove cast shadow for object detection in some surveillance frames. The experiment results are shown in Fig 4 and Fig 5.

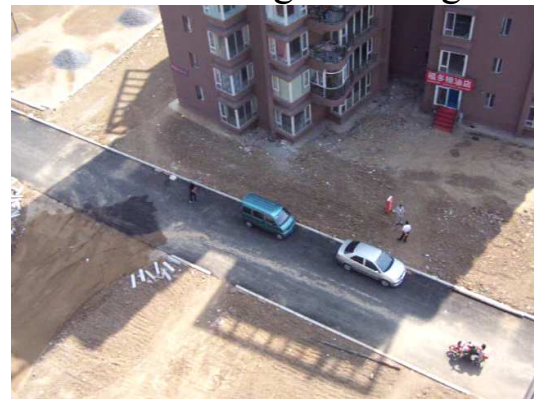

a) current frame

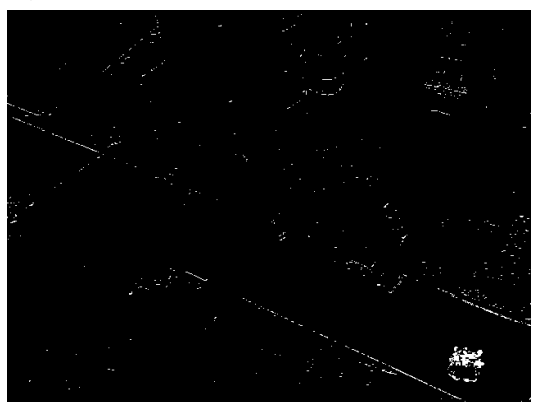

c) result after cast shadow remove

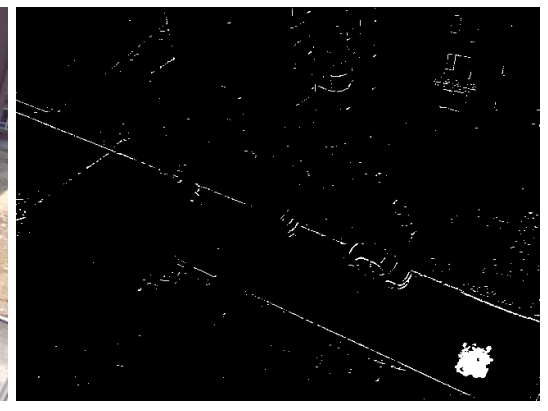

b ) target candidata area

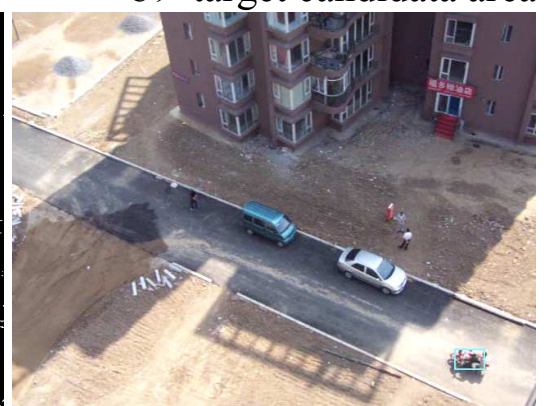

d) target extract

Fig. 4. Removes the cast shadow of motor

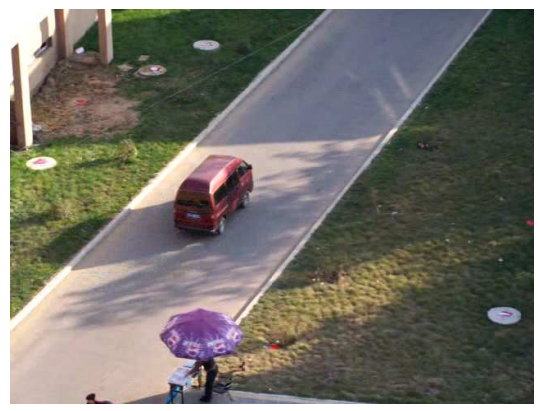

a) current frame

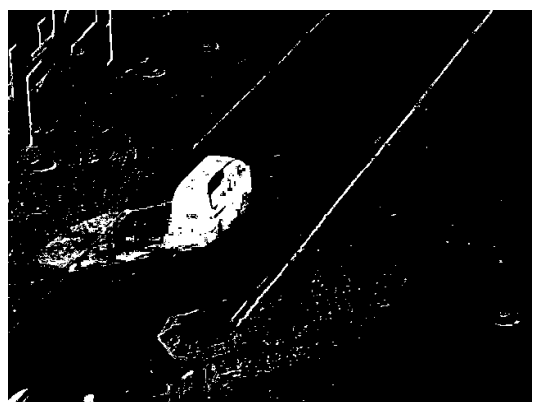

c) result after cast shadow remove

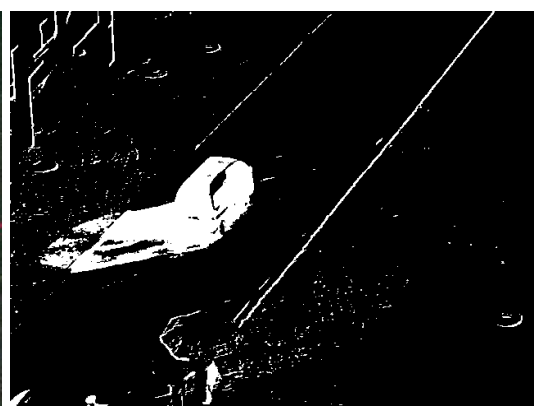

b) target candidata area

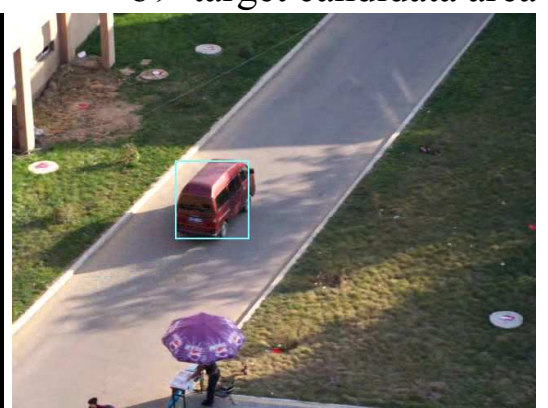

d) target extract

Fig. 5. Removes the cast shadow of a car

Fig 4 (b) and fig 5(b) shown the target candidata area detect results by calculate the difference between current frame and background. From Fig 4 (c) and fig 5(c) we can see that our method can remove the almost cast shadow area from target candidata area. Though there are miss remove pixels around the shadow area because the image model in shadow boundary is more complex, the target still can be extracted correctly from the foreground without cast shadow. 


\section{Conclusions}

In this paper, we propose a method to detect cast shadow of moving object in outdoor surveillance scene based on the tricolor attenuation model. According to the attenuation relationship between each color channel and background model, the cast shadow pixel can be detected by single pixel. By removing the cast shadow area, the real object could be extract corretly. The measure is easy to implement and effiective, can be widely used in outdoor video surveillance system.

\section{Acknowledgement}

This research work is funded by the Natural Science Foundation of China (Grant No. 60835004 and 60871078).

\section{References}

[1] D. C. Knill, D. Kersten, and P.Mamassian, "Geometry of shadows," J. Opt. Soc. Amer. A, vol. 14, no. 12, pp. 3216-3232, 1997.

[2] H. Jiang, and M. Drew, "Tracking objects with shadows," in Proc. ICME03: Int. Conf. Multimedia and Expo, pp. 100-105, 2003.

[3] Y. Wang, K.F. Loe, and J.K. Wu, "Adynamic conditional randomfield model for foreground and shadow segmentation," IEEE Trans on Pattern Analysis and Machine Intelligence., vol. 28, no. 2, pp. 279-289, 2006.

[4] L. Xu, F. H. Qi, and R. J. Jiang, "Shadow removal from a single image," in Proc. 6th Int. Conf. Intelligent Systems Design and Applications, pp. 1049-1054. 2006

[5] E. Salvador, A. Cavallaro, and T. Ebrahimi, "Shadow identification and classification using invariant color models," in Proc. IEEE Int. Conf. Acoustics, Speech, and Signal Processing, pp.1545-1548, 2001.

[6] G. Finlayson, S. Hordley, G. Schaefe, and G. Y. Tian, "Illuminant and device invariant colour using histogram equalization," Pattern Recognition, vol. 38, pp. 179-190, 2005.

[7] A. Leone and C. Distante, "Shadow detection for moving objects based on texture analysis, "Pattern Recognition, vol. 40, 2007, pp. 1222-1233.

[8] K. Barnard and G. Finlayson, "Shadow identification using colour ratios, "Proceedings of the IS\&T/SID Eighth Color Imaging Conference: Color Science,Systems and Applications, 2000, pp. 97-101.

[9] J.Tian, J. Sun, and Y. Tang, "Tricolor attenuation model for shadow detection." IEEE Trans. On Image Processing, vol. 18, no. 10, pp.2355-63, 2009

[10] STAUFFER, C. AND GRIMSON, W. Learning patterns of activity using real time tracking. IEEE Trans on Pattern Analysis and Machine Intelligence 22, 8, 747-767. 2000.

[11] Z.Q.Bian, X.G.Zhang, Pattern Recognition. TSINGHUA UNIVERSITY PRESS. 2004 